Research Article

\title{
Experimental Investigation of the Crater Caused by Hypervelocity Rod Projectile Impacting on Rocks
}

\author{
Yanyu Qiu, ${ }^{1,2}$ Songlin Yue $\mathbb{D}^{2}{ }^{2}$ Mingyang Wang $\mathbb{D}^{1,2}$ Gan Li, ${ }^{1,2}$ Yihao Cheng, \\ Zhangyong Zhao, ${ }^{2}$ and Zhongwei Zhang ${ }^{2}$ \\ ${ }^{1}$ School of Mechanical Engineering, Nanjing University of Science and Technology, Nanjing 210094, China \\ ${ }^{2}$ State Key Laboratory for Disaster Prevention \& Mitigation of Explosion \& Impact, College of Defense Engineering, \\ Army Engineering University of PLA, Nanjing 210007, China
}

Correspondence should be addressed to Songlin Yue; yslseu@hotmail.com and Mingyang Wang; wmyrf@163.com

Received 12 December 2019; Revised 5 April 2020; Accepted 29 June 2020; Published 24 July 2020

Academic Editor: M. I. Herreros

Copyright (c) 2020 Yanyu Qiu et al. This is an open access article distributed under the Creative Commons Attribution License, which permits unrestricted use, distribution, and reproduction in any medium, provided the original work is properly cited.

To investigate the cratering effects of hypervelocity rod projectile impacting on rocks, a two-stage light gas gun was used to carry out 10 groups of small-scale experiments, whose velocity ranges from $1.5 \mathrm{~km} / \mathrm{s}$ to $4.1 \mathrm{~km} / \mathrm{s}$. After each experiment, the morphology and size of the hypervelocity impacting crater were accurately obtained by using a device for image scanning. According to the morphology of the final crater, the impact crater can be divided into crushing area, spallation area, and radial crack area. Based on the experimental results of steel projectile vertical impacting on granite targets, the relationship between the depth and the diameter of the crater is analyzed, i.e., $h / D \approx 0.1 \sim 0.2$; it shows that the depth of the crater is much smaller than the diameter of the crater, and the crater seems to be a shallow dish. The relation between the kinetic energy of the projectile and the size of the crater was discussed. With the increase of the projectile kinetic energy, it is uncertain whether the depth of the crater increases, but the volume of the crater will increase. Lastly, dimensionless analysis of the impact crater was carried out. Specifically, the limitations of point source solutions to hypervelocity rod projectile impact cratering have been proved, and there is no essential difference to calculate the final crater by using the energy scale or the momentum scale.

\section{Introduction}

The problem of hypervelocity impact on rocky media originated from the study of Geology and Planetary Science [1,2]. With the rise of hypervelocity kinetic weapons, the study of such problems began to attract more and more attention [3-5]. The cratering phenomenon is one of the important effects of hypervelocity impact, and the crater size can be used as a bridge to calculate the ground shock effects by establishing equivalent models. Moreover, the impact crater size including depth and diameter is restricted by the hydrodynamic limit of materials. Therefore, it is very important to study the mechanism and propagation process of cratering caused by hypervelocity impact, which is also vital for understanding the damage effect of the hypervelocity kinetic energy weapon.

Generally, hypervelocity impact is a high-temperature, high-pressure, and high strain-rate process caused by the rapid release of energy. It is convenient to divide cratering processes into two stages, i.e., a relatively short initial high-pressure stage and a longer cratering flow stage [6]. In the initial high-pressure stage, a strong shock produced by the hypervelocity impact propagates outward from the source, creating a crushing region. When the high pressure reflects on the surface, it becomes the rarefaction wave which leads to spallation. The crater continues to form after shock crushing and spallation. Melosh [7] clarified the relation between the detached shock and the excavation flow. The initial intensity and the propagation law of the shock wave can be easily and successfully measured by a variety of techniques. However, less success had been achieved in predicting the final crater size. The author found that the excavation flow velocity was a small fraction of the particle velocity, which was sensitive to the constitutive equation of materials. It was difficult to calculate cratering by simplistic constitutive equations. Therefore, experimental investigation based on the physical model and dimensional analysis is necessary to study the problem of hypervelocity impact cratering. 
This cratering problem can be conveniently divided into three regimes. In the "early stage," the impact velocity and the impedances of the two materials determine the shock propagation speed. In the "intermediate stage," the pressures are still large compared to either material strength, viscosity, or gravity-induced stresses so that the response is still much like that of an inviscid, compressible fluid. In the "late stage," the pressures decay down to the kilo bar level or less, where depending upon the problem, material strength, viscosity, or gravity forces act to retard and ultimately stop the crater growth at its final configuration. An interesting phenomenon was discovered that both small-scale impact craters in the laboratory and bowl-shaped craters which are less than $5 \mathrm{~km}$ in diameter on the Earth are controlled by strength (of rocks) [8]. It is reasonable that the processes which lead to the final crater are strongly influenced by low-stress material properties and for large craters, by gravity. In almost all cases, any variable in early and intermediate time regimes can be assumed to be independent of viscosity and gravity. The experiments [9-12] showed that small-scale craters were strength-dominated, whereas large-scale craters were gravity-dominated. Thus, point source assumption is developed and proved to define a single scalar "coupling parameter" measure; let the dimensions of $C$ be given by

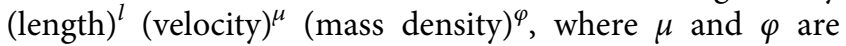
constants for given materials. Note that, with $\mu=2 / 3$ and $\varphi=1 / 3, C$ has the units of (energy) ${ }^{1 / 3}$. With $\mu=-1 / 3$ and $\varphi=1 / 3$, it has the units of (momentum $)^{1 / 3}$. However, a point source solution is to be a limit of solutions as the impactor radius $r_{0} \longrightarrow 0$. Therefore, some researchers considered point source limits for specific materials. The most common is for perfect gases and fluids, where, as already mentioned, the point source solution for one-dimensional spherical explosion as given by Taylor (1950) and Sedov (1946) is now classical. However, to date, there are a large number of different phenomena and results that can be successfully correlated and explained by the concept [6]. Generally, its final utility of applicability must be judged for cases.

At present, there are lots of investigations on the mechanism and propagation process of hypervelocity impact cratering, including results of small-scale experiments in the laboratory and large-scale meteorite impact events in the nature. However, the physical model and dimensional analysis were always established on the spherical projectile, and a lot of studies did not consider cratering caused by the rod projectile. Actually, hypervelocity kinetic energy weapons are always rod projectiles. Besides, it is nearly impossible to build a theoretical model to calculate hypervelocity rod projectile impact cratering with simplistic constitutive equations. Therefore, it is important to investigate the cratering effects caused by the hypervelocity rod projectile, which should be based on small-scale experiments and dimensional analysis.

\section{Experimental Procedure of Hypervelocity Projectile Impacting on Rocks}

A two-stage light gas gun (Figure 1) was used, which can accelerate a projectile whose weight in grams to 3 to $5 \mathrm{~km} / \mathrm{s}$.
The projectile velocity is obtained through the method of using the laser velocimeter, whose effective measuring range is $0.1 \mathrm{~km} / \mathrm{s} \sim 10 \mathrm{~km} / \mathrm{s}$. The target is placed horizontally in an enclosed target chamber. After each experiment, the residual projectile and the target are recovered.

The projectile has an ogive nose shape whose caliber radius head $(\mathrm{CRH})$ equals to 3 , and the diameter and the length are equal to $7.2 \mathrm{~mm}$ and $36 \mathrm{~mm}$, respectively (i.e., the length-diameter ratio is 5). The material is $30 \mathrm{CrMnSiNiA}$, the mass of the projectile is $9.67 \mathrm{~g}$, and the density is $7850 \mathrm{~kg} / \mathrm{m}^{3}$. The projectile is mounted in a polycarbonate sabot with a diameter of $18 \mathrm{~mm}$. The sabot is formed as a three-lobed structure and a mechanical shelling device (Figure 2). The projectile penetrates the target after the sabot is decorticated by the shelling device.

The target material of all experiments is granite. The parameters of granite measured before the experiment are density, elastic P-wave velocity, uniaxial compressive strength, ultimate shear strength, shear modulus, and Poisson's ratio. As shown in Figure 3, granite blocks are enclosed by C30 concrete and placed in a steel barrel. As the wave impedance of C30 concrete is close to that of granite, it is equivalent to increasing the size of the granite target.

The target is made of granite with a density $\left(\rho_{t}\right)$ of $2670 \mathrm{~kg} / \mathrm{m}^{3}$, a longitudinal wave velocity $\left(c_{t}\right)$ of $4900 \mathrm{~m} / \mathrm{s}$, a uniaxial compressive strength $\left(f_{c}\right)$ of $150 \mathrm{MPa}$, a ultimate shear strength $\left(\tau_{s}\right)$ of $1.0 \mathrm{GPa}$, a shear modulus $(G)$ of 27.0 GPa, a Poisson's ratio $(\nu)$ of 0.2 , and Hugoniot elastic limit $(H)$ of $2.36 \sim 2.63 \mathrm{GPa}$. The cross section of the target is a square with a side length of $600 \mathrm{~mm}$ and a total thickness of $800 \mathrm{~mm}$. The target is surrounded by a steel cylinder with an inner diameter of $900 \mathrm{~mm}$ and a wall thickness of $10 \mathrm{~mm}$ (Figure 3).

There were 10 groups of hypervelocity impact experiments conducted, whose velocity range was $1.5 \mathrm{~km} / \mathrm{s} \sim 4.1 \mathrm{~km} / \mathrm{s}$. At the end of each experiment, the recovery of the projectile and the measurement of the target were carried out. In all the experiments, no residual projectile was found; therefore, erosion damage of projectiles occurred. There were no tensile cracks on the edge of the rock target, which indicated that the target was large enough to be considered as a semi-infinite target without being affected by the boundary reflection wave.

\section{Results and Discussion of the Impact Crater}

3.1. The Morphology of the Crater. The concepts of the transient crater and final crater are very important for brittle media such as rocks. Because the tensile strength of the brittle medium is far lower than the compressive strength, the reflected tensile wave on the surface of the target will cause irregular fragmentation of the target, which is called "spalling." If the zone of the crater caused by spalling is excluded, a bowl-shaped crater will be obtained. This part of the crater is formed entirely by the flow of the medium driven by the shock compression wave, which can be called as the "transient crater." Because of the existence of spalling, the diameter and volume of the crater will be greatly increased, and the crater as a whole will present a very rough and irregular shallow dish shape on the inner surface. The 


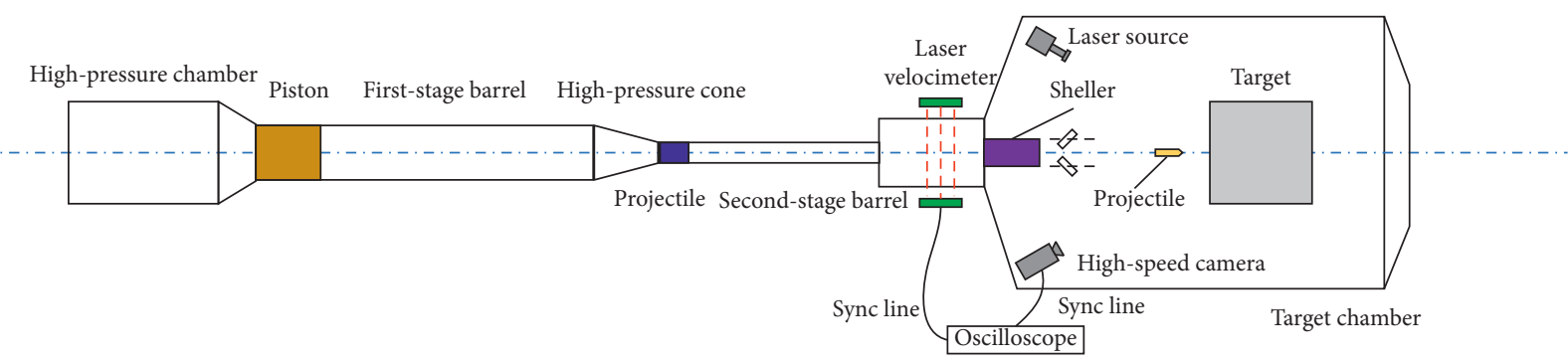

Figure 1: Schematic diagram of the experimental system for a two-stage light gas gun.

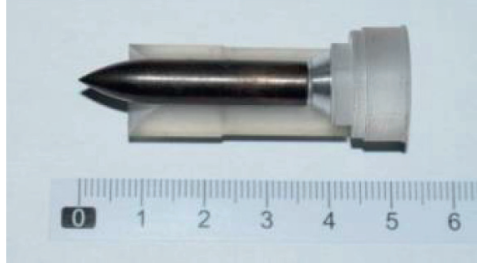

(a)

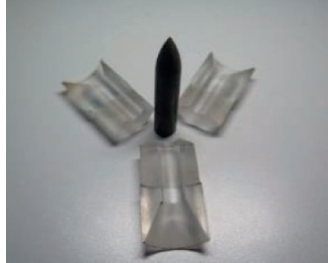

(b)

FIgURE 2: Photographs of the projectile with sabots.

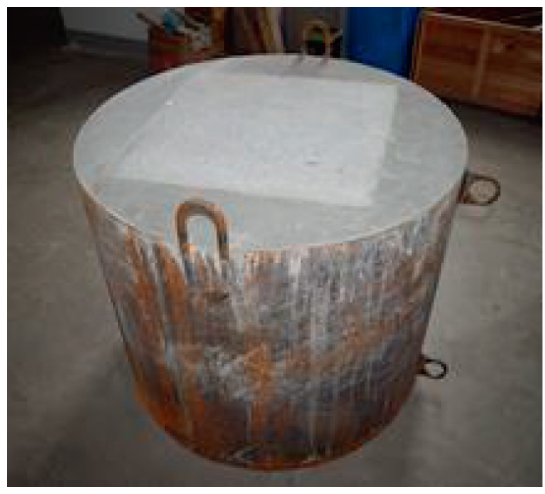

Figure 3: Photograph of targets.

final morphology of the crater affected by shock compression and spalling is called as the "final crater."

By observation of the final crater, it can be found that the impact crater can be divided into three typical zones after the hypervelocity penetration. Each zone owns its obvious characteristics, which are as follows:

(1) The first zone is the crushing area. As it is shown in Figure 4, the center of the crater is concave downward, and there are lots of fine powders at the center of the crater. This results in the fact that the material is intensely compressed, and the original granite particles become fine powders.

(2) The second zone is the spallation area. As shown in Figure 5, spallation is obvious around the concave region at the center of the impact crater. As the tensile strength of granite is much lower than the compressive strength, spallation occurs on the free surface under the action of shock wave propagation and reflection, which also lead to an irregular shape of the impact crater.

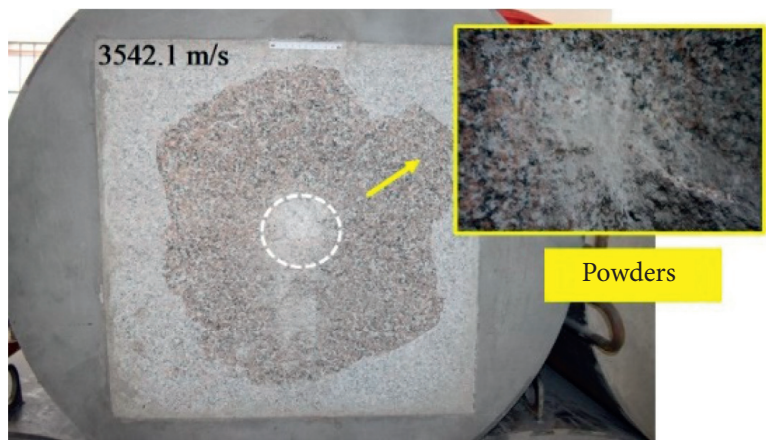

Figure 4: Crushing area at the bottom of the crater.

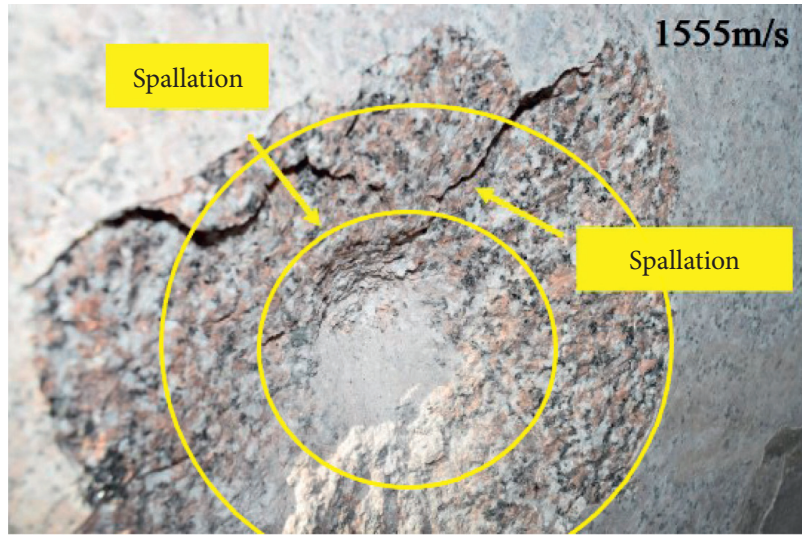

Figure 5: Spallation area around the center of the crater.

(3) The third zone is the radial crack area. When the impact speed is relatively high, radial cracks are distributed radially on the target. As shown in Figure 6, on the edge of the impact crater, obvious radial cracks can be seen before the rock blocks were 


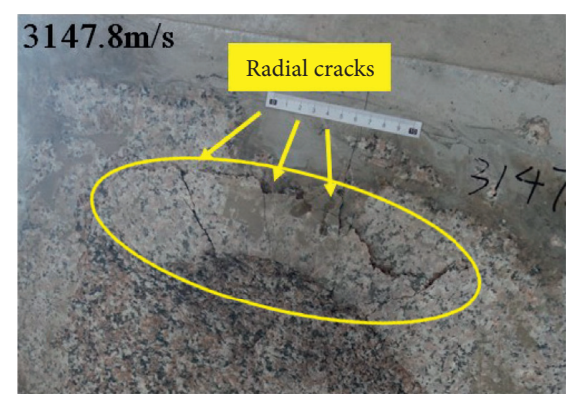

FIGURE 6: Radial cracks on the free surface.

stripped off. As shown in Figure 7, the impact crater is of a regular circular shape initially. While the blocks of the radial crack area were stripped off, the shape of the impact crater is no longer regular, and the diameter increases apparently.

3.2. The Size of the Crater. A device for image scanning of surface morphology was used to measure the crater size, and the scanned image and profile of the crater are shown in Figure 8.

As brittle material can be destroyed and peeled on the free surface by the tension wave, the crater shape is very irregular. Therefore, each target is scanned by three independent cross sections to obtain the maximum depth of the crater and the average diameter of the crater, and the crater volume can be calculated by numerical calculation. The statistics in Table 1 are the experiment results. As shown in Table 1, when the velocity of the projectile is over $2.231 \mathrm{~km} / \mathrm{s}$, the depth of the crater increases with the increase of velocity. The depth of the crater at the velocity of $1.555 \mathrm{~km} / \mathrm{s}$ is greater than that at the velocity of $1.829 \mathrm{~km} / \mathrm{s}$ and $2.231 \mathrm{~km} / \mathrm{s}$. The experimental results are in good agreement with the results of depth inversion in the range of $1.6 \mathrm{~km} / \mathrm{s}$ to $1.8 \mathrm{~km} / \mathrm{s}$. The average diameter of the crater increases with the increase of projectile velocity. The shape of the crater is irregular due to the spallation around the crater. In Table $1, D$ is the diameter of the crater, $h$ is the depth of the crater, and $V$ is the volume of the crater, and all of these parameters are the measurable apparent crater size. At this time, crater is also called the final crater.

According to the experimental results, the relationship between the depth and the diameter of the crater is analyzed, i.e., $h / D \approx 0.1 \sim 0.2$. It shows that the depth of the crater is much smaller than the diameter of the crater, and the crater seems to be a shallow dish. The relationship between normalized diameter and depth is also shown in Figure 9. Besides, the experimental results of hypervelocity projectiles impacting on gabbro by Polanskey and Ahrens [13] are also given, where the projectiles are spherical, and the targets are San Marcos gabbro, and the impact velocity ranges from $1.7 \mathrm{~km} / \mathrm{s}$ to $6.5 \mathrm{~km} / \mathrm{s}$.

Some interesting conclusions can be drawn from Figure 9: (1) the depth of the crater which is caused by the rod projectile (in this article) is 1-2 times of the length of the projectile, while the depth of the crater caused by the spherical projectile is $3-10$ times of the length of the projectile. (2) From the shape of the impact crater, the diameter of the crater caused by the spherical projectile is close to the depth of the crater, while the diameter of the crater caused by the rod projectile (in this paper) is much larger than the depth of the crater. (3) No matter whether the projectile is rod or spherical and no matter whether the target medium is granite or gabbro, the diameter of the crater is much larger than the diameter of the projectile, which is more than 10 times (the prerequisite is that impact velocity is more than $5 \mathrm{Mach}$ ). (4) From the results of the crater, the shape of the crater caused by the spherical projectile is more regular, while the shape of the crater caused by the rod projectile is more discrete.

Due to the limitation of observation and measurement, little is known about the actual situation of transient crater formation. In recent years, MEMIN, a German research team, had carried out a series of hypervelocity impact experiments of tuff, sandstone, and quartzite, which measured the process and morphology of transient cratering. Kenkmann et al. [14] and Dufresne et al. [15] used a three-dimensional laser to measure the inner wall of the crater and considered that the contour of the transient crater can be fitted by a quadratic paraboloid, i.e., the parabola can be determined by the central depression of the crater and the angle of the splash. For the experimental results, parabola was used to fit the central concave part of the crater to get the transient crater. The typical section is shown in Figure 10, which gives the fitting result of the crater at a projectile velocity of $2.231 \mathrm{~km} / \mathrm{s}$.

The parabolic equation for fitting the transient crater in Figure 10 is as follows:

$$
y=0.0155 x^{2}+0.02554 x-45.23118
$$

The volume of the transient crater $\left(V_{t c}\right)$ is as follows:

$$
V_{t c}=\frac{\pi\left(4 a c-b^{2}\right)^{2}}{32 a^{3}},
$$

where, $a, b$, and $c$ are coefficients of the parabolic equation, which can be obtained from equation (1). The results of the final and transient crater under different impact velocities have been listed in Table 2 .

3.3. Relation between the Kinetic Energy of the Projectile and the Size of the Crater. Combining Tables 1 and 2, the kinetic energy of the projectile can be known easily. Then, the relation between the kinetic energy of the projectile and the size of the crater is shown in Figures 11-13.

Figure 11 and Table 2 show that there is little difference in volume of the transient crater and final crater under the same kinetic energy of the projectile when it is less than $40 \mathrm{~kJ}$. However, when the kinetic energy increases, the volume deviates greatly, and the discreteness is large. The results indicate that when the kinetic energy is small, the shape of the impact crater at the bottom of the target was similar to a rotating paraboloid. However, with the increase of the kinetic energy, the shape of the impact crater is no longer regular. Thus, the method of rotating paraboloid 


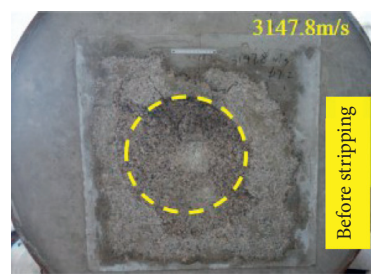

(a)

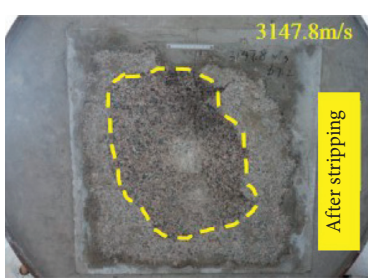

(b)

Figure 7: The influence of stripping on the shape of the crater.

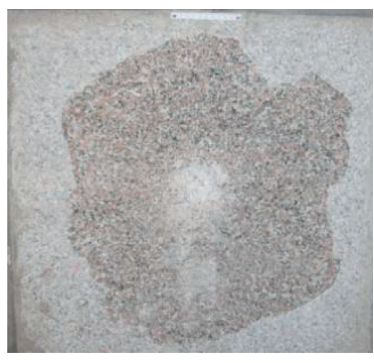

(a)

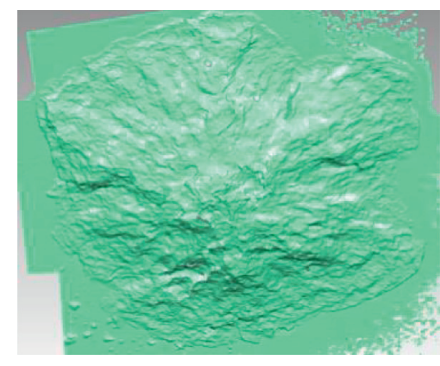

(b)

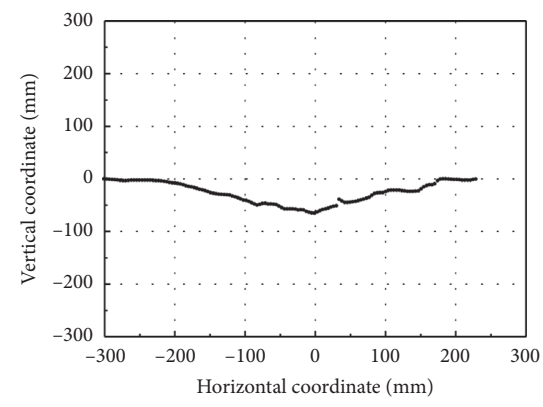

- The profile of the crater

(c)

FIGURE 8: Cratering test on granite under hypervelocity projectile impact. (a) Photograph of a crater. (b) 3D scanned image of the crater. (c) Crater profile.

TABle 1: Experimental results of the final crater.

\begin{tabular}{lccccccc}
\hline No. & \multicolumn{3}{c}{ Parameter of the projectile } & \multicolumn{3}{c}{ Size of the final crater } \\
& $\rho_{p}\left(\mathrm{~g} / \mathrm{cm}^{3}\right)$ & $d_{p}(\mathrm{~cm})$ & $m_{p}(\mathrm{~g})$ & $v_{i}(\mathrm{~km} / \mathrm{s})$ & $D(\mathrm{~cm})$ & $h(\mathrm{~cm})$ & $V\left(\mathrm{~cm}^{3}\right)$ \\
\hline 1 & 7.85 & 0.72 & 9.67 & 1.5550 & 16.7 & 4.770 & 372 \\
2 & 7.85 & 0.72 & 9.67 & 1.8294 & 18.5 & 4.468 & 503 \\
3 & 7.85 & 0.72 & 9.67 & 2.2310 & 27.5 & 4.606 & 652 \\
4 & 7.85 & 0.72 & 9.67 & 2.8069 & 28.5 & 5.137 & 715 \\
5 & 7.85 & 0.72 & 9.67 & 2.8782 & 33.5 & 6.260 & 1575 \\
6 & 7.85 & 0.72 & 9.67 & 3.1478 & 40.5 & 6.068 & 1391 \\
7 & 7.85 & 0.72 & 9.67 & 3.1996 & 38.7 & 5.979 & 1716 \\
8 & 7.85 & 0.72 & 9.67 & 3.5421 & 47.0 & 6.222 & 2863 \\
9 & 7.85 & 0.72 & 9.67 & 3.5584 & 46.1 & 6.584 & 6.67 \\
10 & 7.85 & 0.72 & 9.67 & 4.1356 & 59.7 & 6.565 & 7.55 \\
\hline
\end{tabular}

fitting the transient crater is not suitable for the impact crater caused by the projectile with a large kinetic energy.

In Figure 12, the relation between the kinetic energy of the projectile and the volume of the final crater in logarithmic coordinates is given. As shown, both the experimental results of this article and the experimental results by Lange et al. [16] are in the same fitting curve. It indicates that the volume of the final crater exponentially increased with the increase of the kinetic energy of the projectile, and the power function of the fitting curve has an exponent of 1.2 (mathematical correlation coefficient $R^{2}=0.96$ ). It is obvious that the volume of the impact crater is always increasing with the increase of the impact kinetic energy. For hypervelocity impacting on rocks, it is uncertain whether the depth of the crater increases by improving the kinetic energy of the projectile, but the volume of the crater will increase. This is because the increase in the kinetic energy leads to much more increase in the diameter of the crater. As shown in Figure 13, the kinetic energy of the projectile and the diameter of the final crater (the diameter data are shown in Table 2) satisfy a linear relationship (linear correlation coefficient $R^{2}=0.976$ ).

\section{Dimensionless Analysis of the Impact Crater}

4.1. The Limitations of Point Source Solutions. Dimensional analysis is a method to determine the similarity criteria by using dimension theory. It is a common method to determine the conditions of cratering in studies [17]. For a spherical projectile, Holsapple and Schmidt [6] believe that 


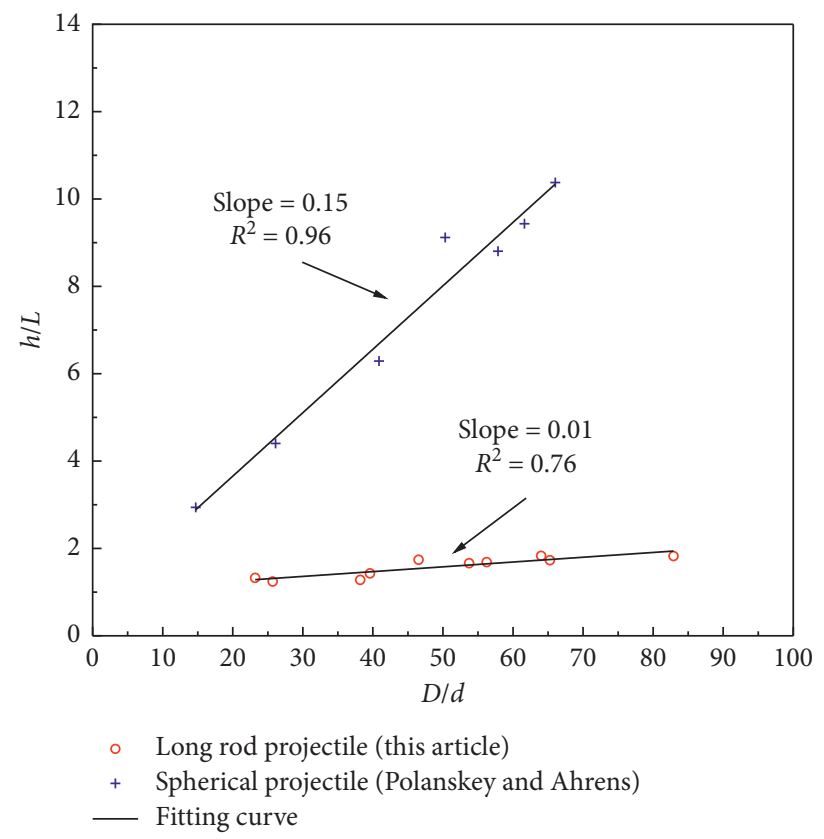

Figure 9: Relation between the dimensionless diameter and the dimensionless depth.

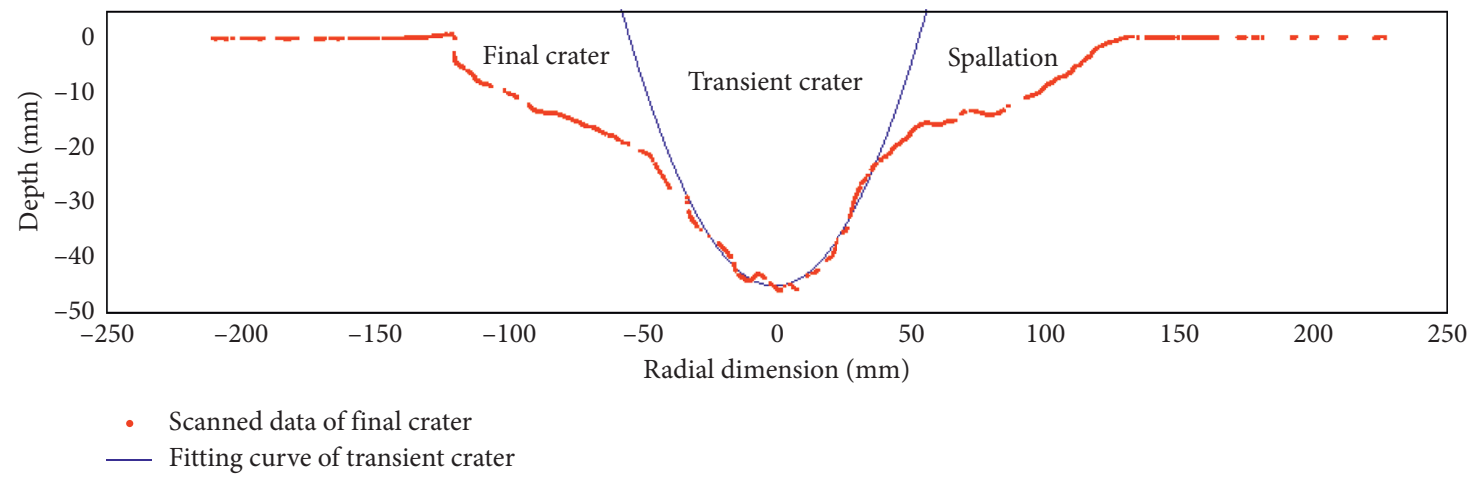

Figure 10: Parabolic fitting of transient craters.

TABLE 2: Experimental results of the transient crater.

\begin{tabular}{lcccccc}
\hline No. & $v_{0}(\mathrm{~m} / \mathrm{s})$ & $V\left(\mathrm{~cm}^{3}\right)$ & $V_{t c}\left(\mathrm{~cm}^{3}\right)$ & $D_{t c}(\mathrm{~cm})$ & $V_{t c} / V$ & $D_{t c} / D$ \\
\hline 1 & 1555.0 & 372 & 105 & 7.6 & 0.28 & 0.46 \\
2 & 1829.4 & 503 & 182 & 10.4 & 0.36 & 0.56 \\
3 & 2231.0 & 652 & 207 & 10.9 & 0.32 & 0.40 \\
4 & 2806.9 & 715 & 288 & 12.6 & 0.40 & 0.44 \\
5 & 2878.2 & 1575 & 248 & 10.1 & 0.16 & 0.30 \\
6 & 3147.8 & 1391 & 376 & 12.7 & 0.27 & 0.31 \\
7 & 3199.6 & 1716 & 320 & 12.5 & 0.19 & 0.32 \\
8 & 3542.1 & 2863 & 1775 & 28.3 & 0.62 & 0.60 \\
9 & 3558.4 & 2761 & 997 & 20.0 & 0.36 & 0.44 \\
10 & 4135.6 & 6299 & - & - & - & - \\
\hline
\end{tabular}

the shape and size of the final crater can be determined by a coupling parameter which can characterize the total kinetic energy or momentum of the projectile. The similar conditions of cratering in hypervelocity impact events can be deduced according to dimensional analysis, which are shown as follows:

$$
V=f\left[\left\{r_{0}, v_{i}, \rho_{p}\right\},\left\{\rho_{t}, Y\right\}, g\right]
$$

where $V$ denotes the crater volume, $r_{0}$ denotes the radius of the projectile, $v_{i}$ denotes the velocity of the projectile, $\rho_{p}$ denotes the density of the projectile, $g$ denotes gravity, $Y$ denotes the strength of the target, and $\rho_{t}$ denotes the density of the target.

Therefore, for any given materials, note parameter $C$ as a coupling parameter. Thus, let the dimensions of $C$ be given by

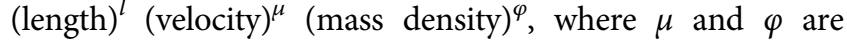
constants for given materials. Note that, with $\mu=2 / 3$ and $\varphi=1 / 3, C$ has the units of (energy) $)^{1 / 3}$. With $\mu=1 / 3$ and $\varphi=1 / 3$, it has the units of (momentum $)^{1 / 3}$ :

$$
C=r_{0} v_{i}^{\mu} \rho_{p}{ }^{\psi} \text {. }
$$

According to the point source theory and power law, equation (3) can be written as follows:

$$
V=f\left[r_{0} v_{i}^{\mu} \rho_{p}^{\psi}, \rho_{t}, Y, g\right],
$$




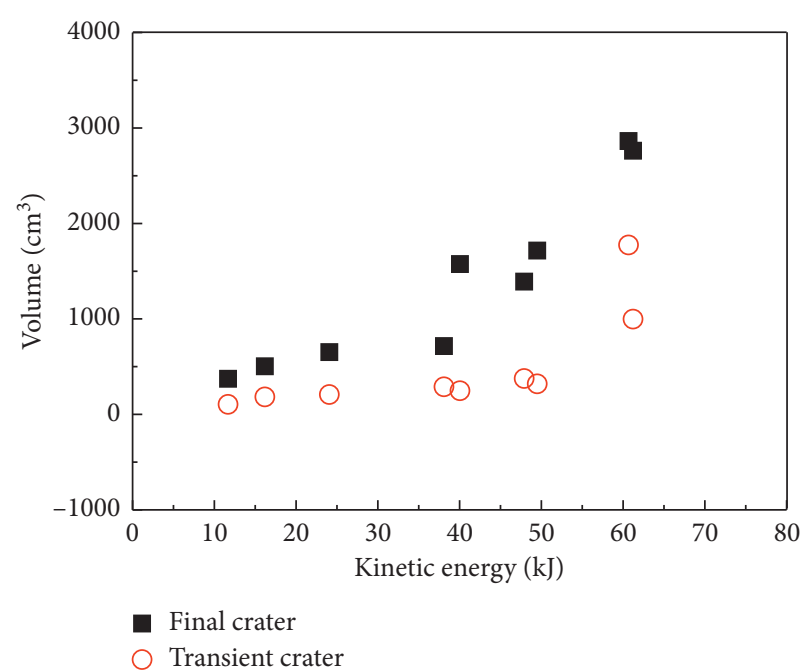

FIgURE 11: The volumes of the final and transient crater change with the kinetic energy of the projectile.

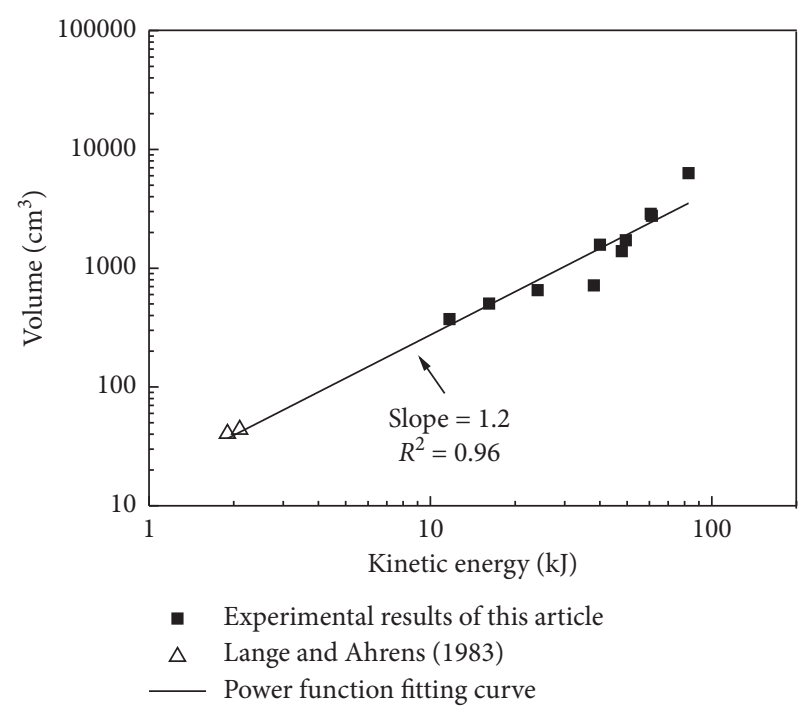

FIGURE 12: Relation between the kinetic energy of the projectile and the volume of the final crater.

where $\mu$ and $\psi$ are constants determined by the experiment. This expression involves five quantities and three independent dimensional units and can therefore be written in terms of two dimensionless variables in various, but equivalent, ways. One such form is

$$
\frac{V \rho_{t}^{3 \varphi}\left(Y / \rho_{t}\right)^{3 \mu / 2}}{\left(r_{0} v_{i}^{\mu} \rho_{p}^{\varphi}\right)^{3}}=f\left[\frac{\left(v_{i}^{2} / g\right)\left(Y / \rho_{t}\right)^{\mu / 2} \rho_{t}^{\varphi}}{r_{0} v_{i}^{\mu} \rho_{p}^{\varphi}}\right] .
$$

Equation (6) can be written as

$$
\frac{V}{r_{0}^{3}}\left(\frac{Y}{\rho_{t} v_{i}^{2}}\right)^{3 \mu / 2}\left(\frac{\rho_{t}}{\rho_{p}}\right)^{3 \psi}=f\left[\frac{v_{i}^{2}}{g r_{0}}\left(\frac{Y}{\rho_{t} v_{i}^{2}}\right)^{\mu / 2}\left(\frac{\rho_{t}}{\rho_{p}}\right)^{\psi}\right] .
$$

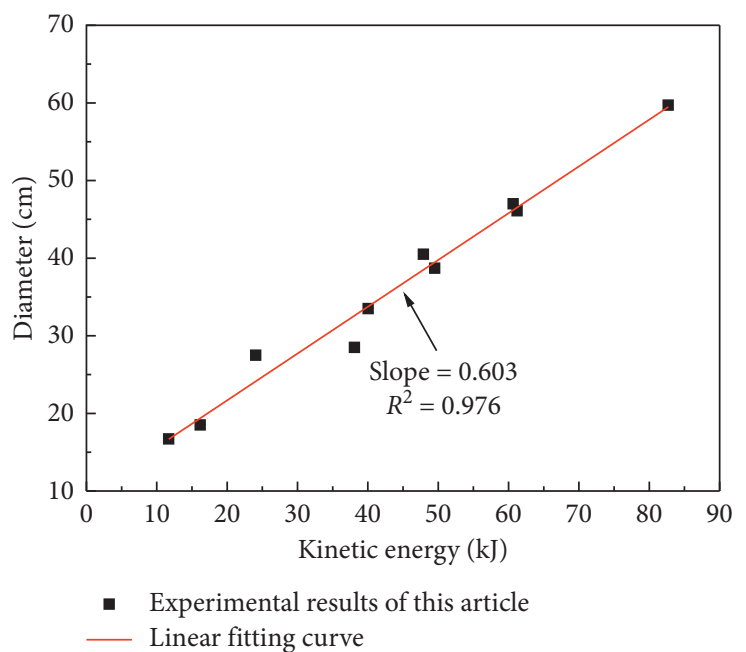

FIGURE 13: Relation between the kinetic energy of the projectile and the diameter of the final crater.

Then, equation (7) can be transformed into

$$
\frac{\rho_{t} V}{m_{p}}\left(\frac{Y}{\rho_{t} v_{i}^{2}}\right)^{3 \mu / 2}\left(\frac{\rho_{t}}{\rho_{p}}\right)^{3 \psi-1}=F\left[\frac{g r_{0}}{v_{i}^{2}}\left(\frac{Y}{\rho_{t} v_{i}^{2}}\right)^{-\mu / 2}\left(\frac{\rho_{t}}{\rho_{p}}\right)^{-\psi}\right] \text {, }
$$

where $m_{p}=\alpha \rho_{p} r_{0}^{3}$, which denotes the mass of the projectile; $F(X)=\alpha^{-1} f\left(X^{-1}\right)$; and $\alpha$ is the coefficient of correction, which is the mass ratio of the irregular projectile to the spherical projectile.

The experiments [9-12] showed that small-scale craters were strength-dominated, whereas sufficiently large craters were gravity-dominated. Therefore, these are strengthdominated craters, and it is reasonable to assume no gravity dependence. Thus, $F(0)$ at the right part of formula (8) is a constant, and then

$$
\frac{\rho_{t} V}{m_{p}} \propto\left(\frac{Y}{\rho_{t} v_{i}^{2}}\right)^{-3 \mu / 2}\left(\frac{\rho_{t}}{\rho_{p}}\right)^{1-3 \psi},
$$

where $\pi_{V}=\left(\rho_{t} V / m_{p}\right)$, which is called crater efficiency, and it is the mass ratio of the crater to the projectile. $\pi_{3}=\left(Y / \rho_{t} v_{i}^{2}\right)$ is called dimensionless strength, and it is the ratio of target material strength to initial dynamic pressure. $\pi_{4}=\left(\rho_{t} / \rho_{p}\right)$ is the density ratio of the target to the projectile, and it is constant for given materials of the projectile and the target. Therefore, equation (9) can be written as

$$
\pi_{V} \propto \pi_{3}^{-3 \mu / 2} \text {. }
$$

There are several models to determine material strength. Housen and Holsapple [18] believed that it is dominated by the friction angle in the Mohr-Coulomb strength model. However, Huirong [19] believed that it can be indicated as uniaxial compressive strength. According to Tobias, Hoertha et al. [20], the strength of the target $(Y)$ was characterized by the uniaxial compressive strength (UCS) for calculation of 
the strength-term $\pi_{3}$. The measured $\mu$-values are located in the theorectical range, i.e., $1 / 3<\mu<2 / 3$. Therefore, it is reasonable to determine material strength by the uniaxial compressive strength. For the almost nonporous granite or gabbro, the product of crater volume and target density, used for calculation of the cratering efficiency $\left(\pi_{V}\right)$, agrees well with the ejected mass. Therefore, the relationship of $\pi_{V}$ and $\pi_{3}$ is shown in Figure 14.

In Figure 14, the relation between normalized crater volume (i.e., crater efficiency) and dimensionless strength is given. The higher the dimensionless strength is, the lower the crater efficiency is. The normalized crater volume ranges from $10^{1}$ to $10^{3}$.

Fitting curves of the impact crater caused by the spherical projectile (i.e., results of Polanskey and Ahrens) and rod projectile (i.e., results of this article) are both in the power-law form as equations (11) and (12), respectively:

$$
\begin{aligned}
& \pi_{V}=0.28 \pi_{3}^{-1.10}, \\
& \pi_{V}=0.18 \pi_{3}^{-1.33} .
\end{aligned}
$$

According to the slope of equations (11) and (12), the coupling parameter $\mu$ equals to 0.71 and 0.89 , respectively, which are not located in the theoretical range $(1 / 3<\mu<2 / 3)$. Both the measured $\mu$-values are greater than $2 / 3$. Therefore, the point source solutions cannot be applicable for the rod projectile.

As stated, the only point source limit that exists is for $r_{0} \longrightarrow 0$ at fixed source energy. For the simple physics of a perfect gas in a one-dimensional problem, one can prove that a point source solution exists by analytical means. For more general materials and for two-dimensional problems such as the cratering problem, there is no actual proof of the existence of such solutions. Generally, the point source solution cannot be used to analyze hypervelocity rod projectile impact cratering.

4.2. The Energy Scale and Momentum Scale of Hypervelocity Impact Cratering. In the problem of cratering caused by the hypervelocity impact of the rod projectile, the "fundamental" independent variables which will affect the dimensional analysis process and the scaling law should be carefully considered. When considering, a projectile of a given material (density $\rho_{p}$ ) and shape (length $L$ and diameter $r_{0}$ ), with mass $m$ and velocity $v_{i}$, impacts at a given target of a given material (density $\rho_{t}$ and strength $Y$ ), with an overlying atmosphere (pressure $P_{a}$ ). The target is assumed to be initially in lithostatic equilibrium under the influence of gravitational field strength $(g)$. In contrast, the choice of the dependent variables is arbitrary, such as the final crater volume $(V)$ or diameter $(R)$.

A simple case occurs for a final crater geometry measure such as the volume $V$. Therefore, it is given by a form such as

$$
V=\phi\left[\left\{L, r_{0}, v_{i}, \rho_{p}\right\},\left\{\rho_{t}, Y\right\}, P_{a}, g\right] .
$$

In a length-force-time system, the independent and dependent variables were developed according to dimensional

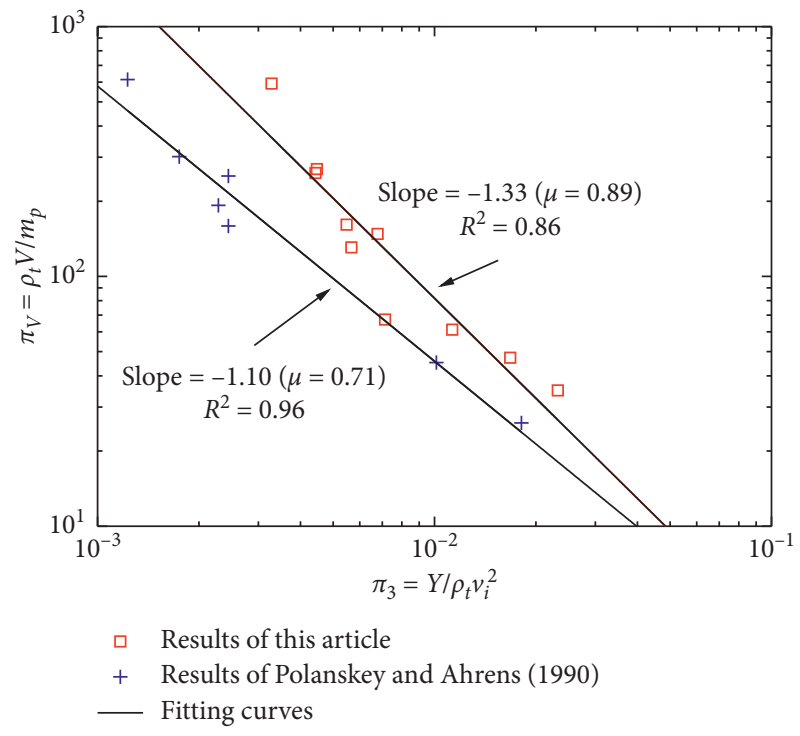

FIGURE 14: The relationship of dimensionless $\pi_{V}$ and $\pi_{3}$.

analysis [21]. This expression involves nine quantities and three independent dimensional units and can therefore be written in terms of six dimensionless variables in various, but equivalent, ways. One such form is

$$
\frac{V}{L r_{0}^{2}}=\phi\left[\left(\frac{\rho_{p} v_{i}^{2}}{Y}\right),\left(\frac{\rho_{p}}{\rho_{t}}\right),\left(\frac{L}{r_{0}}\right),\left(\frac{g L}{v_{i}^{2}}\right),\left(\frac{\rho_{t} g L}{P_{a}}\right)\right] .
$$

4.2.1. The Energy Scale. Equation (14) can be transformed as follows:

$$
\frac{\rho_{p} V}{m_{p}}=\left(\frac{\rho_{p} v_{i}^{2}}{Y}\right)^{\beta} \cdot \Phi\left[\left(\frac{\rho_{p}}{\rho_{t}}\right),\left(\frac{L}{r_{0}}\right),\left(\frac{g L}{v_{i}^{2}}\right),\left(\frac{\rho_{t} g L}{P_{a}}\right)\right],
$$

where $m_{p}=(\pi / 4) \rho_{p} L r_{0}^{2} ; \Pi_{V}=\left(\rho_{p} V / m_{p}\right)$, which is the crater efficiency, and it is the volume ratio of the crater to the projectile; and $\Pi_{Y}=\left(\rho_{p} v_{i}^{2} / Y\right)$, which is the dimensionless strength, and it is the ratio of initial dynamic pressure to material strength; it is obvious that $\Pi_{V}$ and $\Pi_{Y}$ are power exponential relations with an exponent of $\beta$.

Small-scale craters are strength-dominated, whereas sufficiently large craters are gravity-dominated. Therefore, these are strength-dominated craters, and it is reasonable to assume no gravity or atmosphere dependence. In addition, the dimensionless terms $\left(\rho_{p} / \rho_{t}\right)$ and $\left(L / r_{0}\right)$ are constant for given materials of the projectile and the target. Thus, $\Phi[\mathrm{X}]$ at the right part of equation (15) is a constant, and then

$$
\frac{\rho_{p} V}{m_{p}}=\alpha\left(\frac{\rho_{p} v_{i}^{2}}{Y}\right)^{\beta} .
$$

According to the qualitative relationship between the crater efficiency and the dimensionless strength, the lower the strength of the material is, the greater the final crater volume is. Therefore, there must be such conditions: $0<\beta$. Then, it is possible to discuss different forms of expression (16) under different $\beta$-value conditions. 
(1) For $1 \leq \beta$, equation (16) can be rewritten as follows:

$$
1=\frac{m_{p} v_{i}^{2}}{Y \cdot V} \cdot \alpha\left(\frac{\rho_{p} v_{i}^{2}}{Y}\right)^{\beta-1}
$$

Referring to the studies done by Wang et al. and Li and Chen [22, 23], $\Pi_{E}=\left(m_{p} v_{i}^{2} / Y \cdot V\right), 43$ which is widely known as the dimensionless impact factor or dimensionless energy factor. It denotes that the final crater volume increases with the increase of the kinetic energy of the projectile and the decrease of material strength [24, 25].

(2) For $0<\beta<1$, equation (16) can be rewritten as follows:

$$
1=\frac{m_{p} v_{i}^{2}}{Y \cdot V} \cdot \alpha\left(\frac{Y}{\rho_{p} v_{i}^{2}}\right)^{1-\beta}
$$

From equation (18), the final crater volume can also be expressed by the kinetic energy of the projectile and the material strength $[26,27]$.

Therefore, no matter what the $\beta$-value is, the final crater can always be described by the dimensionless impact factor or dimensionless energy factor. If the scaling law, expressed as (17) or (18), is named as the energy scale, then what form is the momentum scale?

4.2.2. The Momentum Scale. Certainly, equation (14) can also be transformed as follows:

$$
\frac{\rho_{t} V}{m_{p}}=\left(\frac{\rho_{t} v_{i}^{2}}{Y}\right)^{\beta} \cdot \Phi\left[\left(\frac{\rho_{p}}{\rho_{t}}\right),\left(\frac{L}{r_{0}}\right),\left(\frac{g L}{v_{i}^{2}}\right)\right],
$$

where $\pi_{V}=\left(\rho_{t} V / m_{p}\right)$ and $\pi_{Y}=\left(\rho_{t} v_{i}^{2} / Y\right)$. Similarly, it is easy to simplify equation (19) as follows:

$$
\frac{\rho_{t} V}{m_{p}}=\alpha\left(\frac{\rho_{t} v_{i}^{2}}{Y}\right)^{\beta}
$$

The same as the above, there must be such conditions: $0<\beta$. Then, it is possible to discuss different forms of expression (20) under different $\beta$-value conditions [28, 29].

(1) For $0.5 \leq \beta$, equation (20) can be rewritten as follows:

$$
1=\frac{m_{p} v_{i}}{V \sqrt{\rho_{t} Y}} \cdot \alpha\left(\frac{\rho_{t} v_{i}^{2}}{Y}\right)^{\beta-0.5}
$$

where $\Pi_{M}=\left(m_{p} v_{i} / V \sqrt{\rho_{t} Y}\right)$, which is named as the dimensionless momentum factor. It denotes that the final crater volume increases with the increase of momentum of the projectile and the decrease of material strength and material density.

(2) For $0<\beta<0.5$, equation (20) can be rewritten as follows:

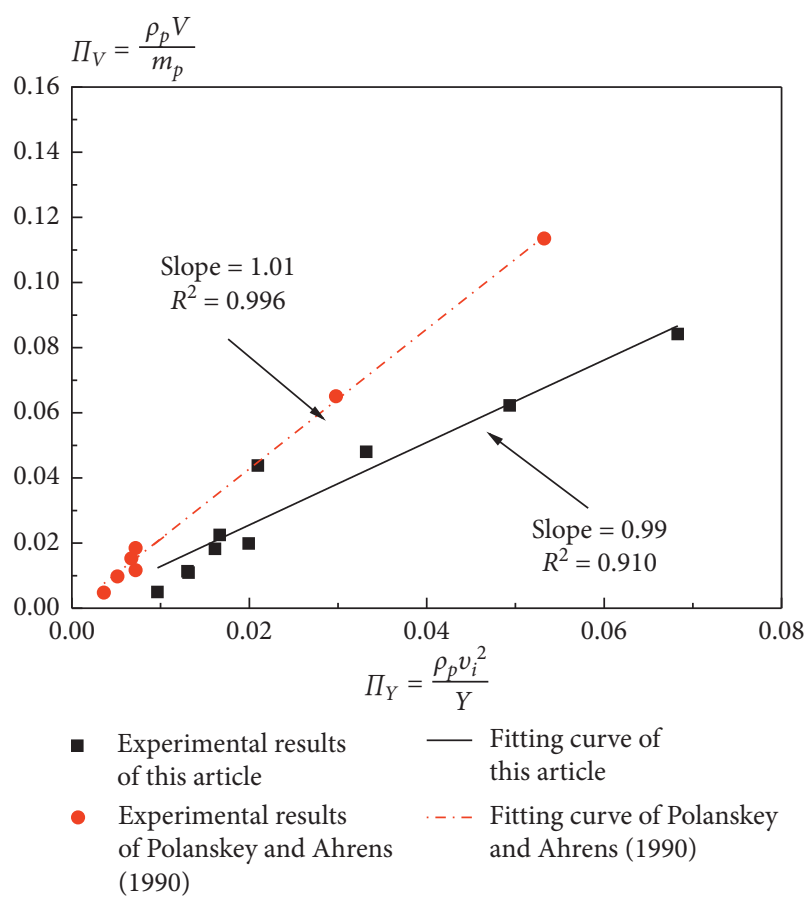

Figure 15: The relation between dimensionless $\Pi_{V}$ and $\Pi_{Y}$ based on the energy scale.

$$
1=\frac{m_{p} v_{i}}{V \sqrt{\rho_{t} Y}} \cdot \alpha\left(\frac{Y}{\rho_{t} v_{i}^{2}}\right)^{0.5-\beta} .
$$

Therefore, no matter what the $\beta$-value is, the final crater can always be described by the dimensionless momentum factor.

4.2.3. The Equivalent Effect. Generally, equation (16) can be regarded as the energy-scale expression, which is described by dimensionless $\Pi_{V}$ and $\Pi_{Y}$. And equation (20) can be regarded as the momentum-scale expression, which is described by dimensionless $\pi_{V}$ and $\pi_{Y}$.

The relation between energy scale and momentum scale can be built up as follows:

$$
\Pi_{M}=\sqrt{\Pi_{E} \cdot \Pi_{m}}=\sqrt{\frac{m_{p} v_{i}^{2}}{Y V} \frac{m_{p}}{\rho_{t} V}}=\frac{m_{p} v_{i}}{V \sqrt{\rho_{t} Y}},
$$

where $\quad \Pi_{m}=\left(m_{p} / \rho_{t} V\right), \quad \Pi_{M}=m_{p} v_{i} / V \sqrt{\rho_{t} Y}, \quad$ and $\Pi_{E}=m_{p} v_{i}^{2} / Y V$. Thus, it is obvious that the dimensionless energy factor and dimensionless momentum factor are not mutually independent; in contrast, they can be converted to each other. Therefore, there is an equivalent effect by using the energy scale or the momentum scale.

According to equation (16), it is easy to establish the relation between dimensionless $\Pi_{V}$ and $\Pi_{Y}$ based on the energy scale, which is shown in Figure 15. In the same way, according to equation (20), it is easy to establish the relation 


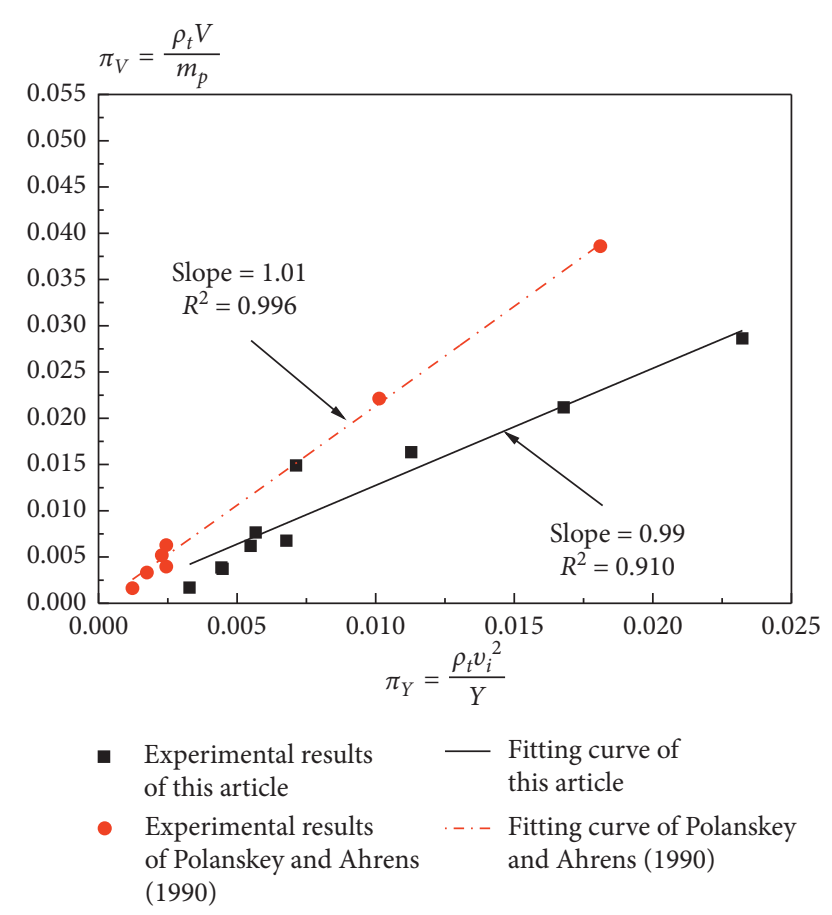

Figure 16: The relation between dimensionless $\pi_{V}$ and $\pi_{Y}$ based on the momentum scale.

between dimensionless $\pi_{V}$ and $\pi_{Y}$ based on the momentum scale, which is shown in Figure 16.

From Figure 15, the fitting curves of the relation between dimensionless $\Pi_{V}$ and $\Pi_{Y}$ are shown as follows:

$$
\begin{cases}\Pi_{V}=1.25 \Pi_{Y}^{0.99}\left(R^{2}=0.910\right), & \text { this article, } \\ \Pi_{V}=2.20 \Pi_{Y}^{1.01}\left(R^{2}=0.996\right), & \text { Polanskey and Ahrens. }\end{cases}
$$

From Figure 16, the fitting curves of the relation between dimensionless $\pi_{V}$ and $\pi_{Y}$ are shown as follows:

$$
\begin{cases}\pi_{V}=1.25 \pi_{Y}^{0.99}\left(R^{2}=0.910\right), & \text { this article, } \\ \pi_{V}=2.20 \pi_{Y}^{1.01}\left(R^{2}=0.996\right), & \text { Polanskey and Ahrens. }\end{cases}
$$

Combining Figures 15 and 16 and equations (24) and (25), it can be concluded that the relation between dimensionless $\Pi_{V}$ and $\Pi_{Y}$ based on the energy scale are the same as the relation between dimensionless $\pi_{V}$ and $\pi_{Y}$ based on the momentum scale. In dimensional analysis of hypervelocity impact cratering, it is equivalent to calculate the final crater by using the energy scale or the momentum scale.

\section{Conclusion}

(1) According to the morphology of the crater, the impact crater can be divided into crushing area, spallation area, and radial crack area.

(2) When a hypervelocity rod projectile impacts on a granite target, spallation occurs on the free surface under the action of shock wave propagation and reflection, which also lead to an irregular shape of the impact crater. It shows that the depth of the crater is much smaller than the diameter of the crater, and the crater seems to be a shallow dish. According to the experimental results, the relationship between the depth and the diameter of the crater is analyzed, i.e., $h / D \approx 0.1 \sim 0.2$.

(3) With the increase of the projectile kinetic energy, it is uncertain whether the depth of the crater increases, but the volume of the crater will increase. This is because the increase of the kinetic energy leads to much more increase of the diameter of the crater.

(4) The volume of the transient and final crater increases with the increase of the projectile kinetic energy, and the contribution of spallation to the volume is growing more rapidly.

(5) When calculating the relationship between dimensionless crater efficiency and dimensionless strength by the dimensional analysis method, the point source solution cannot be used to analyze the problem of cratering caused by the hypervelocity rod projectile. Dimensional analysis was redesigned, and the similarity law was re-established. Another interesting and reasonable conclusion had been proved by experimental investigation in which it is equivalent to calculate the final crater by using the energy scale or the momentum scale.

\section{Data Availability}

The data used to support the findings of this study are included within the article.

\section{Conflicts of Interest}

The authors declare that there are no conflicts of interest regarding the publication of this paper.

\section{Acknowledgments}

The authors acknowledge the financial support received from the Natural Science Foundation of China (Grant nos. 51808552, 51808553, and 11602303), the China Postdoctoral Science Foundation (Grant nos. 2017M621752 and 2018M643853), and the Natural Science Foundation of Jiangsu Province (Grant no. BK20190570).

\section{References}

[1] W. Herrmann and J. S. Wilbeck, "Review of hypervelocity penetration theories," International Journal of Impact Engineering, vol. 5, no. 1, pp. 307-322, 1986.

[2] H. J. Melosh, Impact Cratering, Oxford University Press, New York, NY, USA, 1989.

[3] D. E. Gault and E. D. Heitowit, "The partition of energy for hypervelocity impact craters formed in rock," Sixth Hypervelocity Impact Symposium, Cleveland Ohio, vol. 2, pp. 219546, 1963.

[4] T. H. Antoun, L. A. Glenn, O. R. Walton, P. Goldstein, I. N. Lomov, and B. Liu, "Simulation of hypervelocity 
penetration in limestone," International Journal of Impact Engineering, vol. 33, no. 1-12, pp. 45-52, 2006.

[5] L. I. Zheng, Y. Liu, M. Hu et al., "Damage effect evaluation of God stick space-based kinetic energy weapons," Journal of Vibration and Shock, vol. 35, no. 18, pp. 159-180, 2016.

[6] K. A. Holsapple and R. M. Schmidt, "Point source solutions and coupling parameters in cratering mechanics," Journal of Geophysical Research, vol. 92, no. B7, pp. 6350-6376, 1987.

[7] H. J. Melosh, "Impact cratering mechanics: relationship between the shock wave and excavation flow," Icarus, vol. 62, no. 2, pp. 339-343, 1985.

[8] T. J. Ahrens, K. Xia, and D. Cokert, "Depth of cracking beneath impact craters: new constraint for impact velocity," in AIP Conference Proceedings, Zacatecas, Mexico, July 2002.

[9] K. A. Holsapple and R. M. Schmidt, "On the scaling of crater dimensions: 1. Explosive processes," Journal of Geophysical Research, vol. 85, no. B12, pp. 7247-7256, 1980.

[10] K. A. Holsapple and R. M. Schmidt, "On the scaling of crater dimensions: 2. Impact processes," Journal of Geophysical Research, vol. 87, no. B3, pp. 1849-1870, 1982.

[11] K. A. Holsapple, "The scaling of impact processes in planetary sciences," Annual Review of Earth and Planetary Sciences, vol. 21, no. 1, pp. 333-373, 1993.

[12] K. R. Housen, R. M. Schmidt, and K. A. Holsapple, "Crater ejecta scaling laws-Fundamental forms based on dimensional analysis," Journal of Geophysical Research, vol. 88, no. 17, pp. 2485-2499, 1983.

[13] C. A. Polanskey and T. J. Ahrens, "Impact spallation experiments: fracture patterns and spall velocities," Icarus, vol. 87, no. 1, pp. 140-155, 1990.

[14] T. Kenkmann, K. Wünnemann, A. Deutsch, M. H. Poelchau, F. Schäfer, and K. Thoma, "Impact cratering in sandstone: the MEMIN pilot study on the effect of pore water," Meteoritics \& Planetary Science, vol. 46, no. 6, pp. 890-902, 2011.

[15] A. Dufresne, M. H. Poelchau, T. Kenkmann et al., "Crater morphology in sandstone targets: the MEMIN impact parameter study," Meteoritics \& Planetary Science, vol. 48, no. 1, pp. 50-70, 2013.

[16] M. A. Lange, T. J. Ahrens, and M. B. Boslough, "Impact cratering and spall failure of gabbro," Icarus, vol. 58, no. 3, pp. 383-395, 1984.

[17] J. K. Dienes and J. M. Walsh, "Theory of impact: some general principles and the method of Eulerian codes," High-velocity Impact Phenomenon, pp. 50-61, Academic Press, New York, NY, USA, 1970.

[18] K. R. Housen and K. A. Holsapple, "Ejecta from impact craters," Icarus, vol. 211, no. 1, pp. 856-875, 2011.

[19] A. Huirong, Shock-Induced Damage in Rocks: Application to Impact Cratering, pp. 5-33, California Institute of Technology, Pasadena, CA, USA, 2006.

[20] T. F. Hoerth, J. Hupfer, O. Millon, and M. Wickert, "Momentum transfer in hypervelocity impact experiments on rock targets," Procedia Engineering, vol. 103, pp. 197-204, 2015.

[21] S. L. Schäfer and Y. Y. Qiu, "Modelling experiment methods for cratering effects of explosions in rocks and comparative analysis," Chinese Journal of Rock Mechanics and Engineering, vol. 33, no. 9, 2014.

[22] M. Wang, Y. Qiu, and S. Yue, "Similitude laws and modeling experiments of explosion cratering in multi-layered geotechnical media," International Journal of Impact Engineering, vol. 117, pp. 32-47, 2018.

[23] Q. M. Li and X. W. Chen, "Dimensionless formulae for penetration depth of concrete target impacted by a non- deformable projectile," International Journal of Impact Engineering, vol. 28, no. 1, pp. 93-116, 2003.

[24] C. E. Anderson Jr., D. L. Littlefield, and J. D. Walker, "Longrod penetration, target resistance, and hypervelocity impact," International Journal of Impact Engineering, vol. 14, no. 1-4, pp. 1-12, 1993.

[25] H. Wu, L.-L. Chen, and Q. Fang, "Stability analyses of the mass abrasive projectile high-speed penetrating into concrete target. Part I: engineering model for the mass loss and noseblunting of ogive-nosed projectiles," Acta Mechanica Sinica, vol. 30, no. 6, pp. 933-942, 2014.

[26] H. Wu, Q. Chen, and L.-L. He, "Stability analyses of the mass abrasive projectile high-speed penetrating into concrete target. Part II: structural stability analyses," Acta Mechanica Sinica, vol. 30, no. 6, pp. 943-955, 2014.

[27] G. Ben-Dor, T. A. Dubinsky, and T. Elperin, "High-speed penetration modeling and shape optimization of the projectile penetrating into concrete shields\#," Mechanics Based Design of Structures and Machines, vol. 37, no. 4, pp. 538-549, 2009.

[28] Y. Peng, Q. Fang, H. Wu et al., "Theoretical analyses for terminal ballistic of the projectiles with different nose geometries penetrating into concrete targets," Binggong Xuebao/ Acta Armamentarii, vol. 35, pp. 128-134, 2014.

[29] C. S. Meyer, "Modeling experiments of hypervelocity penetration of adobe by spheres and rods," Procedia Engineering, vol. 58, pp. 138-146, 2013. 